International Journal of Modern Physics A Vol. 32, No. 36 (2017) 1799001 (19 pages) (c) World Scientific Publishing Company DOI: $10.1142 / \mathrm{S} 0217751 \mathrm{X} 17990019$

\title{
Cumulative author index Volume 32
}

\author{
Abdujabbarov, A., see Hakimov \\ Abe, Y., Horikoshi, M. \& Kawamura, \\ Y., Dark energy from gravitational \\ corrections \\ Abreu, E. M. C. \& Neves, M. J., \\ Strong interaction model in DFR \\ noncommutative space-time \\ Abreu, E. M. C., Neto, J. A., Barboza \\ Jr., E. M. \& Nunes, R. C., Tsallis \\ and Kaniadakis statistics from the \\ viewpoint of entropic gravity for- \\ malism \\ Abreu, E. M. C., see Neves \\ Abu-Ajamieh, F., Houtz, R. \& Zheng, \\ R., Phenomenology of bulk scalar \\ singlets in the Randall-Sundrum \\ model
}

Acernese, F., Adams, T., Agatsuma, K., Aiello, L., Allocca, A., Amato, A., Antier, S., Arnaud, N., Ascenzi, S., Astone, P., Bacon, P., Bader, M. K. M., Baldaccini, F., Ballardin, G., Barone, F., Barsuglia, M., Barta, D., Basti, A., Bawaj, M., Bazzan, M., Bejger, M., Belahcene, I., Bersanetti, D., Bertolini, A., Bitossi, M., Bizouard, M. A., Bloemen, S., Boer, M., Bogaert, G., Bondu, F., Bonnand, R., Boom, B. A., Boschi, V., Bouffanais, Y., Bozzi, A., Bradaschia, C., Branchesi, M., Briant, T., Brillet, A., Brisson, V., Bulik, T., Bulten, H. J., Buskulic, D., Buy, C., Cagnoli, G., Calloni, E., Canepa, M., Canizares, P., Capocasa, E., Carbognani, F., Casanueva Diaz, J., Casentini, C., Cavalier, F., Cavalieri, R., Cella, G., Cerboni Baiardi, L., Cerretani, G., Cesarini, E., Chassande-

\begin{tabular}{|c|c|}
\hline 32 (2017) 1750116 & $\begin{array}{l}\text { Mottin, E., Chincarini, A., Chi- } \\
\text { ummo, A., Christensen, N., Chua, } \\
\text { S., Ciolfi, R., Cirone, A., Cleva, F., }\end{array}$ \\
\hline 32 (2017) 1750037 & $\begin{array}{l}\text { Coccia, E., Cohadon, P.-F., Colla, } \\
\text { A., Conti, L., Cortese, S., Coulon, } \\
\text { J.-P., Cuoco, E., D'Antonio, S., }\end{array}$ \\
\hline 32 (2017) 1750099 & $\begin{array}{l}\text { Dattilo, V., Davier, M., Degallaix, } \\
\text { J., De Laurentis, M., Deléglise, } \\
\text { S., Del Pozzo, W., De Rosa, R., } \\
\text { Di Fiore, L., Di Giovanni, M., } \\
\text { Di Girolamo, T., Di Lieto, A., }\end{array}$ \\
\hline 32 (2017) 1750028 & Di Pace, S., Di Palma, I., Di \\
\hline 32 (2017) 1750190 & $\begin{array}{l}\text { Renzo, F., Dolique, V., Ducrot, M., } \\
\text { Fafone, V., Farinon, S., Ferrante, } \\
\text { I., Ferrini, F., Fidecaro, F., Fiori, } \\
\text { I., Fiorucci, D., Flaminio, R., }\end{array}$ \\
\hline 32 (2017) 1750113 & $\begin{array}{l}\text { Fournier, J.-D., Frasca, S., Fras- } \\
\text { coni, F., Frey, V., Gammaitoni, L., } \\
\text { Garufi, F., Gemme, G., Genin, E., } \\
\text { Gennai, A., Germain, V., Ghosh, } \\
\text { A., Ghosh, S., Giazotto, A., Gon- } \\
\text { zalez Castro, J. M., Gosselin, M., } \\
\text { Gouaty, R., Grado, A., Granata, } \\
\text { M., Greco, G., Groot, P., Grun- } \\
\text { ing, P., Guidi, G. M., Harms, } \\
\text { J., Heidmann, A., Heitmann, H., } \\
\text { Hello, P., Hemming, G., Hoak, } \\
\text { D., Hofman, D., Huet, D., In- } \\
\text { tini, G., Isac, J.-M., Jacqmin, T., } \\
\text { Jaranowski, P., Jonker, R. J. G., } \\
\text { Kéfélian, F., Khan, I., Koley, S., } \\
\text { Kowalska, I., Królak, A., Kuty- } \\
\text { nia, A., Lartaux-Vollard, A., Laz- } \\
\text { zaro, C., Leaci, P., Leonardi, M., } \\
\text { Leroy, N., Letendre, N., Loren- } \\
\text { zini, M., Loriette, V., Losurdo, } \\
\text { G., Lumaca, D., Majorana, E., } \\
\text { Maksimovic, I., Man, N., Man- } \\
\text { tovani, M., Marchesoni, F., Mar- } \\
\text { ion, F., Martelli, F., Martellini, L., } \\
\text { Masserot, A., Mastrogiovanni, S., }\end{array}$ \\
\hline
\end{tabular}


Meidam, J., Merzougui, M., Metzdorff, R., Mezzani, F., Michel, C., Milano, L., Miller, A., Minazzoli, O., Minenkov, Y., Moggi, A., Mohan, M., Montani, M., Mours, B., Nardecchia, I., Naticchioni, L., Nelemans, G., Neri, M., Nichols, D., Nissanke, S., Nocera, F., Palomba, C., Paoletti, F., Paoli, A., Pasqualetti, A., Passaquieti, R., Passuello, D., Patricelli, B., Pedurand, R., Perreca, A., Piccinni, O. J., Pichot, M., Piergiovanni, F., Pillant, G., Pinard, L., Poggiani, R., Popolizio, P., Porter, E. K., Prodi, G. A., Punturo, M., Puppo, P., Rapagnani, P., Razzano, M., Regimbau, T., Rei, L., Ricci, F., Robinet, F., Rocchi, A., Rolland, L., Romano, R., Rosińska, D., Ruggi, P., Salconi, L., Sassolas, B., Schmidt, P., Sentenac, D., Sequino, V., Sieniawska, M., Singhal, A., Sorrentino, F., Stratta, G., Swinkels, B. L., Tacca, M., Tiwari, S., Tonelli, M., Travasso, F., Tringali, M. C., Trozzo, L., Tsang, K. W., van Bakel, N., van Beuzekom, M., van den Brand, J. F. J., Van Den Broeck, C., van der Schaaf, L., van Heijningen, J. V. \& Vardaro, M., Status of the Advanced Virgo gravitational wave detector

Adams, T., see Acernese

Addazi, A., (Anti)evaporation of dyonic black holes in stringinspired dilaton $f(R)$-gravity

Addazi, A., Brane bounce from logarithmic entropic corrections in the bulk

Addazi, A., Quantum chaos inside black holes

Adorno, T. C., Gavrilov, S. P. \& Gitman, D. M., Exactly solvable cases in QED with $t$-electric potential steps

Agatsuma, K., see Acernese

Ahmadov, A. I., A study of the process $e^{+}+e^{-} \rightarrow e^{+}+e^{-}+$ $p+\bar{p}$ by the two-photon mechanism $\gamma \gamma \rightarrow p \bar{p}$ at high energies

Ahuja, G. \& Sharma, S., Texture zero mass matrices and $\mathrm{CP}$ violation

Aiello, L., see Acernese
32 (2017) 1744003 32 (2017) 1744003

32 (2017) 1750102

32 (2017) 1750174

32 (2017) 1750087

32 (2017) 1750105 32 (2017) 1744003

32 (2017) 1750104

32 (2017) 1742005

32 (2017) 1744003
Akeroyd, A. G., Moretti, S., Yagyu, K. \& Yildirim, E., Light charged Higgs boson scenario in 3-Higgs doublet models

Akhavan, A., Gauge symmetry breaking in gravity and auxiliary effective action

Alam, S., Behera, S., Kumar, S. \& Sahoo, S., Constraining capability of $Z \gamma h$ production at the ILC

Aleedaneshvar, A. \& Khorramian, A., The impact of CMS inclusive jet data on parton distribution functions and their uncertainties

32 (2017) 1750145

32 (2017) 1750026

32 (2017) 1750017

Ali, A. F., see Faizal

Ali, A. F., see Sepehri

Ali, A., Precision tests of the Standard Model: Rare $B$-meson decays

Allocca, A., see Acernese

Allotey, F. K. A., Prof. Abdus Salam, my teacher and mentor: The role of ICTP in Africa

Almeida, G. M. A., see Santos

Alsaleh, S., Thermodynamics of BTZ black holes in gravity's rainbow

Alves, D. W. F., Hoyos, C., Nastase, H. \& Sonnenschein, J., Knotted solutions, from electromagnetism to fluid dynamics

32 (2017) 1750200

32 (2017) 1744003

Amato, A., see Acernese

Amooshahi, M., Canonical relativistic quantization of electromagnetic field in the presence of an anisotropic conductor magnetodielectric medium

Anacleto, M. A., Brito, F. A., Holanda, O. \& Passos, E., Induction of the Lorentz-violating effective actions in quantum electrodynamics

Andrić, I., Jonke, L., Jurman, D. \& Nielsen, H. B., Dynamical and quenched random matrices and homolumo gap

Anjana, V., Harikumar, E. \& Kapoor, A. K., Noncommutative spacetime and Hausdorff dimension

Antier, S., see Acernese

Antoniadis, I., Scale hierarchies and string cosmology

32 (2017) 1741006

32 (2017) 1750146

32 (2017) 1750076

32 (2017) 1750209

32 (2017) 1750128

32 (2017) 1750046

32 (2017) 1750183

32 (2017) 1744003

32 (2017) 1730012

Antusch, S., Cazzato, E. \& Fischer, O., Sterile neutrino searches at future $e^{-} e^{+}, p p$ and $e^{-} p$ colliders 
Cumulative author index

Aoki, Y., Aoyama, T., Bennett, E., Kurachi, M., Maskawa, T., Miura, K., Nagai, K.-i., Ohki, H., Rinaldi, E., Shibata, A., Yamawaki, K. \& Yamazaki, T. (LatKMI Collaboration), Lattice study of the scalar and baryon spectra in many-flavor QCD

Aoki, Y., Aoyama, T., Bennett, E., Kurachi, M., Maskawa, T., Miura, K., Nagai, K.-i., Ohki, H., Rinaldi, E., Shibata, A., Yamawaki, K. \& Yamazaki, T. (LatKMI Collaboration), Topological insights in many-flavor QCD on the lattice

Aoyama, T., see Aoki

Aoyama, T., see Aoki

Arash, F., see Momeni-Feili

Arnaud, N., see Acernese

Arraut, I., The Nambu-Goldstone theorem in nonrelativistic systems

Arroyo-Ureña, M. A., Díaz, E., Meza-Aldama, O. \& TavaresVelasco, G., $\tau^{-} \rightarrow$ $\ell_{i}^{-} \ell_{i}^{+} \ell_{j}^{-} \bar{\nu}_{j} \nu_{\tau}$ decays with a magnetic dipole term

Asadi, A., Mehraban, H. \& Shahryari, Z., Three-body decays of $D_{s}^{+} \rightarrow \eta\left(\eta^{\prime}\right) \pi^{+} \pi^{0}$

Ascenzi, S., see Acernese

Astone, P., see Acernese

Athenodorou, A., Bennett, E., Bergner, G. \& Lucini, B., Recent results from $\mathrm{SU}(2)$ with one adjoint Dirac fermion

Azizi, A. \& Nasirimoghadam, S., Behavior of light polarization in photon-scalar interaction

Bacon, P., see Acernese

Bader, M. K. M., see Acernese

Baeta Scarpelli, A. P., Mariz, T., Nascimento, J. R. \& Petrov, A. Yu., On the anomalies in Lorentz-breaking theories (Erratum)

Bai, S., see Bian

Bai, S., see Su

Bai, S., see Wang

Bai, S., see Wang

Bai, X.-Z., see Chen

Baker, D., Kodwani, D., Pen, U.-L. \& Yang, I-S., A self-consistency check for unitary propagation of Hawking quanta

Bakke, K., see Mota

Baldaccini, F., see Acernese
32 (2017) 1747010

32 (2017) 1747005 32 (2017) 1747005

32 (2017) 1747010 32 (2017) 1750036 32 (2017) 1744003

32 (2017) 1750127

32 (2017) 1750195

32 (2017) 1750110 32 (2017) 1744003 32 (2017) 1744003

32 (2017) 1747006

32 (2017) 1750177

32 (2017) 1744003

32 (2017) 1744003

32 (2017) 1792002 32 (2017) 1746009 32 (2017) 1746005 32 (2017) 1746006 32 (2017) 1746004 32 (2017) 1750207

32 (2017) 1750198 32 (2017) 1750140 32 (2017) 1744003
Ballardin, G., see Acernese

Bamba, K., Large-scale magnetic fields, non-Gaussianity, and gravitational waves from inflation

Banerjee, D., Maji, P. \& Sahoo, S., Study of the rare decays $B_{s, d}^{*} \rightarrow$ $l^{+} l^{-}$in $Z^{\prime}$ model

Banerjee, G. \& Panda, B., F-string solution in $\mathrm{AdS}_{4} \times \mathcal{C} \mathcal{P}^{3} p p$ wave background

Banerjee, R., Gangopadhyay, S. \& Mukherjee, P., On the question of symmetries in nonrelativistic diffeomorphism-invariant theories

Banerjee, R., see Mitra

Barboza Jr., E. M., see Abreu

Bardakci, K., Reminiscences on Stanley Mandelstam

Barone, F., see Acernese

Barsuglia, M., see Acernese

Barta, D., see Acernese

Bartoš, E., Dubnička, S., Dubničková, A. Z. \& Hayashii, H., The mass and width differences of the neutral and charged $\rho(770)$, $\rho(1450)$ and $\rho(1700)$ mesons from $e^{+} e^{-} \rightarrow \pi^{+} \pi^{-}$and $\tau^{-} \rightarrow \nu_{\tau} \pi^{-} \pi^{0}$ processes

Basak, S., see Goswami

Basti, A., see Acernese

Basu, A., Simplifying the one-loop five graviton amplitude in type IIB string theory

Batalin, I. A. \& Lavrov, P. M., On quantum-mechanical equations of motion in representation dependent of external sources

Battista, E., Esposito, G. \& Dell'Agnello, S., On the foundations of general relativistic celestial mechanics

Bawaj, M., see Acernese

Bazeia, D., Lima, E. E. M. \& Losano, L., Kinks and branes in models with hyperbolic interactions

Bazhutov, Yu. N., Vereshkov, G. M. \& Kuksa, V. I., Experimental and theoretical premises of new stable hadron existence

Bazzan, M., see Acernese

Behera, S., see Alam

Bejger, M., see Acernese

Bekaert, X. \& Skvortsov, E. D., Elementary particles with continuous spin
32 (2017) 1744003

32 (2017) 1747021

32 (2017) 1750075

32 (2017) 1750005

32 (2017) 1750115

32 (2017) 1750210

32 (2017) 1750028

32 (2017) 1740006

32 (2017) 1744003

32 (2017) 1744003

32 (2017) 1744003

32 (2017) 1750154

32 (2017) 1750059

32 (2017) 1744003

32 (2017) 1750074

32 (2017) 1750189

32 (2017) 1730022

32 (2017) 1744003

32 (2017) 1750163

32 (2017) 1750188

32 (2017) 1744003

32 (2017) 1750017

32 (2017) 1744003

32 (2017) 1730019 
Belahcene, I., see Acernese Belich, H., see Louzada

Belich, H., see Mota

Belitsky, A. V., Leading order analysis of twist-3 space- and time-like cut vertices in QCD

Belli, P., Guest Editor's Preface to the Special Issue on low background techniques

Benchikha, A. \& Merad, M., Energydependent harmonic oscillator in noncommutative space: A path integral approach

Bennai, M., see Mounzi

Bennett, E., see Aoki

Bennett, E., see Aoki

Bennett, E., see Athenodorou

Berera, A., Stanley Mandelstam my graduate supervisor

Bergner, G., see Athenodorou

Berkovits, N., Scientific biography of Stanley Mandelstam: 1981-2016

Bernabei, R. \& Incicchitti, A., Low background techniques in $\mathrm{NaI}(\mathrm{Tl})$ setups

Bernal, N., Heikinheimo, M., Tenkanen, T., Tuominen, K. \& Vaskonen, V., The dawn of FIMP Dark Matter: A review of models and constraints

Bernstein, J. G., see Heckman

Berra-Montiel, J., del Río, E. \& Molgado, A., Polysymplectic formulation for topologically massive Yang-Mills field theory

Bersanetti, D., see Acernese

Bertolini, A., see Acernese

Bertone, G., see Bozorgnia

Beylin, V., Bezuglov, M. \& Kuksa, V., Analysis of scalar Dark Matter in a minimal vectorlike Standard Model extension

Bezuglov, M., see Beylin

Bhat, M., see Monteiro

Bhowmick, S. \& Chatterjee, S., Towards timelike singularity via AdS dual

Bian, L., Chen, N. \& Jiang, Y., Higgs pair production in the $\mathrm{CP}$-violating two-Higgs-doublet model

Bian, T., Gao, J., Zhang, C., Cui, X.-H., Wang, Y.-W., Bai, S., Su, F., Wang, D., Cai, Y.-H. \& Koratzinos, M., CEPC booster design study
32 (2017) 1744003 32 (2017) 1750010 32 (2017) 1750140

Bian, T., see Su

Bian, T., see Wang

Biekötter, A., Hewett, J. L., Kim, J. S., Krämer, M., Rizzo, T. G., Rolbiecki, K., Tattersall, J. \&

32 (2017) 1730018 Weber, T., Complementarity of resonant scalar, vector-like quark and superpartner searches in eluci-

32 (2017) 1702001 dating new phenomena

Bilić, N., Dimitrijevic, D. D., Djordjevic, G. S. \& Milosevic, M. Tachyon inflation in an AdS braneworld with backreaction

32 (2017) 1750194 32 (2017) 1750119 32 (2017) 1747005 32 (2017) 1747010 32 (2017) 1747006

32 (2017) 1740009 32 (2017) 1747006

32 (2017) 1740011

32 (2017) 1743007

32 (2017) 1730023

32 (2017) 1750133

32 (2017) 1750101 32 (2017) 1744003 32 (2017) 1744003 32 (2017) 1730016

32 (2017) 1750042 32 (2017) 1750042 32 (2017) 1750021

32 (2017) 1750122

32 (2017) 1746002

32 (2017) 1746009
Botelho, L. C. L., Studies on Polyakov and Nambu-Goto random surface path integrals on $\operatorname{QCD}(S U(\infty))$ : Interquark potential and phenomenological scattering amplitudes

Bischer, I., Grandou, T. \& Hofmann, R., Massive loops in thermal SU(2) Yang-Mills theory: Radiative corrections to the pressure beyond two loops

Bitossi, M., see Acernese

Biyajima, M., see Mizoguchi

Bizouard, M. A., see Acernese

Bloemen, S., see Acernese

Boer, M., see Acernese

Bogaert, G., see Acernese

Boiko, R. S., Chemical purification of lanthanides for low-background experiments

Bondarenko, A. I., see Markov

Bondu, F., see Acernese

Bonnand, R., see Acernese

Boom, B. A., see Acernese

Boos, E., Bunichev, V., Dudko, L. \& Perfilov, M., Modeling of anomalous $W t b$ interactions in single top quark events using subsidiary fields

Bornman, N., de Mello Koch, R. \& Tribelhorn, L., Rotating restricted Schur polynomials

Boroun, G. R., Rezaei, B. \& Heidari, S., Nuclear longitudinal structure function in $e A$ processes at the $\mathrm{LHeC}$

Boschi, V., see Acernese

Botelho, L. C. L., QCD $(S U(\infty))$ as a model of infinite-dimensional constant gauge field configurations

Bouffanais, Y., see Acernese
32 (2017) 1746005

32 (2017) 1746006

32 (2017) 1750032

32 (2017) 1750039

32 (2017) 1750118

32 (2017) 1744003

32 (2017) 1750057

32 (2017) 1744003

32 (2017) 1744003

32 (2017) 1744003

32 (2017) 1744003

32 (2017) 1743005

32 (2017) 1750144

32 (2017) 1744003

32 (2017) 1744003

32 (2017) 1744003

32 (2017) 1750008

32 (2017) 1750150

32 (2017) 1750197

32 (2017) 1744003

32 (2017) 1750031

32 (2017) 1750030

32 (2017) 1744003 
Cumulative author index

Bozhilov, P., String solutions in $\mathrm{AdS}_{3} \times S^{3} \times S^{3} \times S^{1}$ with $B$-field

Bozorgnia, N. \& Bertone, G., Implications of hydrodynamical simulations for the interpretation of direct dark matter searches

Bozzi, A., see Acernese

Bradaschia, C., see Acernese

Branchesi, M., see Acernese

Brandt, F. T., Chishtie, F. A. \& McKeon, D. G. C., The effective potential in nonconformal gauge theories

Briant, T., see Acernese

Brillet, A., see Acernese

Brink, L., Stanley Mandelstam and me and life on the light-cone

Brisson, V., see Acernese

Brito, F. A., see Anacleto

Brower, R. C., see Hasenfratz

Bufalo, R. \& Ghasemkhani, M., Three-dimensional noncommutative Yukawa theory: Induced effective action and propagating modes

Bufalo, R., Pimentel, B. M. \& Soto, D. E., Normalizability analysis of the generalized quantum electrodynamics from the causal point of view

Bulik, T., see Acernese

Bulten, H. J., see Acernese

Bunichev, V., see Boos

Buskulic, D., see Acernese

Buy, C., see Acernese

Bär, O., Chiral perturbation theory and nucleon-pion-state contaminations in lattice $\mathrm{QCD}$

Băloi, M.-A. \& Crucean, C., Fermion production by a dependent of time electric field in de Sitter universe

Cadoni, M. \& Jain, P., How is the presence of horizons and localized matter encoded in the entanglement entropy?

Cagnoli, G., see Acernese

Cai, Y.-H., see Bian

Calloni, E., see Acernese

Campos, S. D., see Okorokov

Canepa, M., see Acernese

Canizares, P., see Acernese

Cannoni, M., Lorentz invariant relative velocity and relativistic binary collisions

Capocasa, E., see Acernese

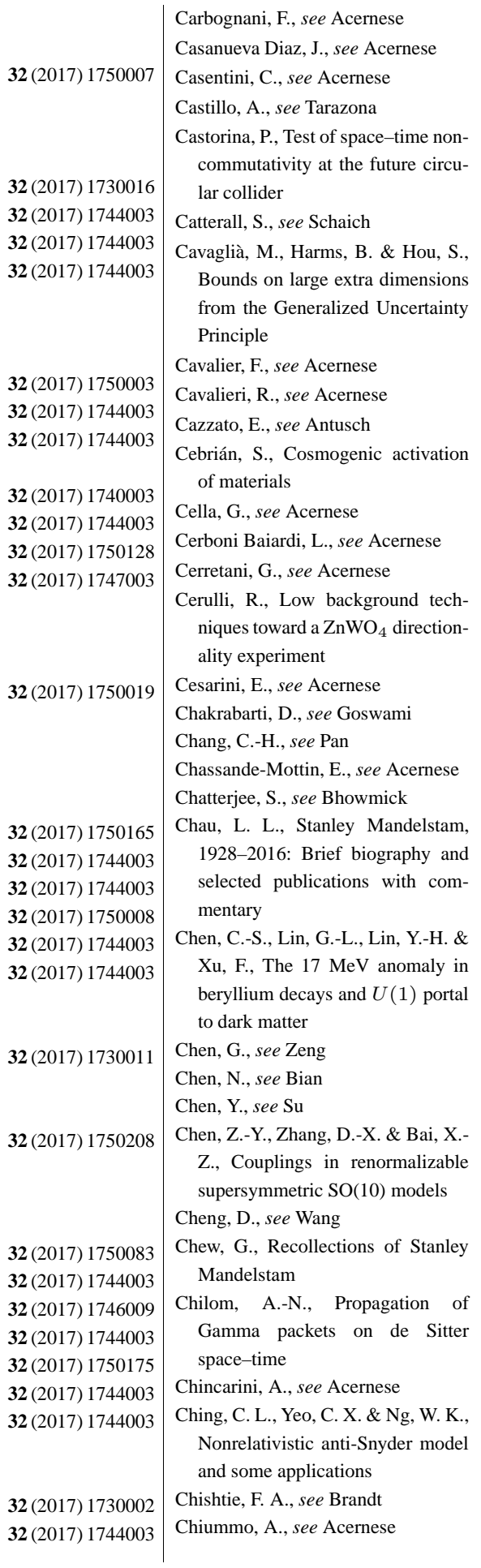

32 (2017) 1744003

32 (2017) 1744003

32 (2017) 1744003

32 (2017) 1750050

32 (2017) 1750179

32 (2017) 1747019

32 (2017) 1750082

32 (2017) 1744003

32 (2017) 1744003

32 (2017) 1750078

32 (2017) 1743006

32 (2017) 1744003

32 (2017) 1744003

32 (2017) 1744003

32 (2017) 1743009

32 (2017) 1744003

32 (2017) 1750059

32 (2017) 1750067

32 (2017) 1744003

32 (2017) 1750122

32 (2017) 1740012

32 (2017) 1750178

32 (2017) 1750124

32 (2017) 1746002

32 (2017) 1746005

32 (2017) 1750207

32 (2017) 1746008

32 (2017) 1740001

32 (2017) 1750167

32 (2017) 1744003

32 (2017) 1750009

32 (2017) 1750003

32 (2017) 1744003 
Chivukula, R. S., Simmons, E. H. \& Vignaroli, N., The jet energy profile: A BSM analysis tool

Chivukula, R. S., see Simmons

Choudhury, D. K., Saikia, B. \& Kalita, K., Momentum fractions carried by quarks and gluons in models of proton structure functions at small $x$

Christensen, N., see Acernese

Chua, S., see Acernese

Chung, W. S., see Shababi

Ciolfi, R., see Acernese

Cioroianu, E.-M., Rich gauge structures from a unitary approach of some massless gauge fields of spins one and two

Cirone, A., see Acernese

Clavelli, L., Six indications of radical new physics in supernovae Ia

Cleva, F., see Acernese

Coccia, E., see Acernese

Cohadon, P.-F., see Acernese

Colla, A., see Acernese

Conti, L., see Acernese

Copia, L., see Nisi

Cortese, S., see Acernese

Cortés, J. L. \& López-Sarrión, J., Fine-tuning problems in quantum field theory and Lorentz invariance: A scalar-fermion model with a physical momentum cutoff

Coulon, J.-P., see Acernese

Cox, P., Medina, A. D., Ray, T. S. \& Spray, A., Implications of diphoton searches for a radion in the bulk-Higgs scenario

Crucean, C., see Băloi

Cui, X., see Wang

Cui, X.-H., see Bian

Cui, Z.-F., see Pan

Cuoco, E., see Acernese

D'Antonio, S., see Acernese

Dafinei, I., see Nisi

Dahiya, H. \& Randhawa, M., Electromagnetic and axial-vector form factors of the quarks and nucleon

Dai, D.-C., Serious limitations of the strong equivalence principle

Danevich, F. A., Radiopure tungstate and molybdate crystal scintillators for double beta decay experiments

Das, A., see Sidharth

Das, A., see Sidharth

Das, S., see Faizal
32 (2017) 1747022 32 (2017) 1747023

32 (2017) 1750107 32 (2017) 1744003 32 (2017) 1744003 32 (2017) 1750170 32 (2017) 1744003

32 (2017) 1750044 32 (2017) 1744003

32 (2017) 1750181 32 (2017) 1744003 32 (2017) 1744003 32 (2017) 1744003 32 (2017) 1744003 32 (2017) 1744003 32 (2017) 1743003 32 (2017) 1744003

32 (2017) 1750084 32 (2017) 1744003

32 (2017) 1750020 32 (2017) 1750208 32 (2017) 1746006 32 (2017) 1746009 32 (2017) 1750067 32 (2017) 1744003 32 (2017) 1744003 32 (2017) 1743003

32 (2017) 1750185

32 (2017) 1750068

32 (2017) 1743008 32 (2017) 1750117 32 (2017) 1750173 32 (2017) 1750049
Datta, A., see Rashed

Dattilo, V., see Acernese

Davier, M., see Acernese

Davydov, A., Sveshnikov, K. \& Voronina, Yu., Vacuum energy of one-dimensional supercritical Dirac-Coulomb system de Cruz Pérez, J., see Solà

de Gracia, G. B., Spin jumping in the context of a QCD effective model

de Lacroix, C., Erbin, H., Kashyap, S. P. \& Sen, A. \& Verma, M., Closed superstring field theory and its applications

De Laurentis, M., see Acernese

de Medeiros Varzielas, I., Rasmussen, R. W. \& Talbert, J., Bottom-up discrete symmetries for cabibbo mixing

de Mello Koch, R., see Bornman

De Rosa, R., see Acernese

Degallaix, J., see Acernese

Del Pozzo, W., see Acernese

del Río, E., see Berra-Montiel

Delbourgo, R., Life with Salam (1959-1976)

Deleidi, L., see Salesi

Dell'Agnello, S., see Battista

Deléglise, S., see Acernese

Deng, G.-M. \& Huang, Y.-C., $Q-\Phi$ criticality and microstructure of charged AdS black holes in $f(R)$ gravity

Di Fiore, L., see Acernese

Di Giovanni, M., see Acernese

Di Girolamo, T., see Acernese

Di Lieto, A., see Acernese

Di Pace, S., see Acernese

Di Palma, I., see Acernese

Di Renzo, F., see Acernese

Di Vacri, M. L., see Nisi

Di Vecchia, P., My interaction with Stanley

Di, H., see Yang

Diaz, R. A., see Tarazona

Dil, E., Can quantum black holes be $(q, p)$-fermions?

Dillon, B. M., Han, C., Lee, H. M. \& Park, M., KK graviton resonance and cascade decays in warped gravity

Dimitrijevic, D. D., see Bilić

Ding, R., Huang, L., Li, T. \& Zhu, B., Interpreting $750 \mathrm{GeV}$ diphoton excess with R-parity violating
32 (2017) 1750060

32 (2017) 1744003

32 (2017) 1744003

32 (2017) 1750054

32 (2017) 1730014

32 (2017) 1750041

32 (2017) 1730021

32 (2017) 1744003

32 (2017) 1750047

32 (2017) 1750150

32 (2017) 1744003

32 (2017) 1744003

32 (2017) 1744003

32 (2017) 1750101

32 (2017) 1741005

32 (2017) 1750086

32 (2017) 1730022

32 (2017) 1744003

32 (2017) 1750204

32 (2017) 1744003

32 (2017) 1744003

32 (2017) 1744003

32 (2017) 1744003

32 (2017) 1744003

32 (2017) 1744003

32 (2017) 1744003

32 (2017) 1743003

32 (2017) 1740019

32 (2017) 1750051

32 (2017) 1750050

32 (2017) 1750080

32 (2017) 1745006 32 (2017) 1750039 
Cumulative author index

supersymmetry

Djordjevic, G. S., see Bilić

Dolique, V., see Acernese

Dong, Z.-J., see Zeng

Dremin, I. M., Elastic scattering and spatial inelastic profiles of high energy protons

Du, Y.-L., see Luo

Dubnička, S., see Bartoš

Dubničková, A. Z., see Bartoš

Ducrot, M., see Acernese

Dudko, L., see Boos

Duff, M. J., Abdus Salam at Imperial College

Díaz, E., see Arroyo-Ureña

Edmonds, D., Farrah, D., Ho, C. M., Minic, D., Ng, Y. J. \& Takeuchi, T., Testing Modified Dark Matter with galaxy clusters: Does dark matter know about the cosmological constant?

Ellis, J., Where is particle physics going?

Englert, C., Li, Q., Spannowsky, M., Wang, M. \& Wang, L., VBS $W^{ \pm} W^{ \pm} H$ production at the HL-LHC and a $100 \mathrm{TeV} p p$ collider

Erbin, H., see de Lacroix

Escobar, C. A. \& Urrutia, L. F., Extended Nambu models: Their relation to gauge theories

Eslami, P., see Mottaghizadeh

Esposito, G., see Battista

Estabar, T. \& Mehraban, H., Analysis of four-body decay of $D$ meson

Fafone, V., see Acernese

Fagundes, D. A., Menon, M. J. \& Silva, P. V. R. G., Leading components in forward elastic hadron scattering: Derivative dispersion relations and asymptotic uniqueness

Faizal, M., Ali, A. F. \& Das, S., Discreteness of time in the evolution of the universe

Faizal, M., see Sepehri

Fan, W., Luo, X. \& Zong, H.-S., Mapping the QCD phase diagram with susceptibilities of conserved charges within NambuJona-Lasinio model

Fanchiotti, H., García Canal, C. A. \& Vento, V., Multiphoton annihilation of monopolium
32 (2017) $1750014 \mid$ Fang, S., see Zheng

32 (2017) 1750039 Fang, Y., Kumar, M., Mellado, B., 32 (2017) 1744003 Zhang, Y. \& Zhu, M., Impact of 32 (2017) 1750124 additional bosons on the exploration of the Higgs boson at the LHC

32 (2017) 1750073 Farahmand, M., Mohammadzadeh, 32 (2017) 1750040 H. \& Mehri-Dehnavi, H., Quan32 (2017) 1750154 tum thermodynamics and quantum 32 (2017) 1750154 entanglement entropies in an ex32 (2017) 1744003 32 (2017) 1750008

32 (2017) 1741001 32 (2017) 1750195 panding universe

Farinon, S., see Acernese

Farrah, D., see Edmonds

Faux, M., The conformal hyperplet

Ferrante, I., see Acernese

Ferricha-Alami, M., see Mounzi

Ferrini, F., see Acernese

Fidecaro, F., see Acernese

Finkelstein, R. J., On the $\operatorname{SLq}(2)$ extension of the standard model and the concept of charge

Fiori, I., see Acernese

32 (2017) 1746001

Fiorucci, D., see Acernese

Fischer, O., see Antusch

Flaminio, R., see Acernese

Floquet, S., Trindade, M. A. S. \& Vianna, J. D. M., Lie algebras and generalized thermal coherent states

32 (2017) 173002

Fodor, Z., Holland, K., Kuti, J., Mondal, S., Nogradi, D. \& Wong,

32 (2017) $1750077 \quad$ C. H., Status of a minimal compos-

32 (2017) 1750065

32 (2017) 1730022

32 (2017) 1750011

32 (2017) 1744003

32 (2017) 1750049 32 (2017) 1750025 te Higgs theory

Fornal, B., Is there a sign of new physics in beryllium transitions?

Fournier, J.-D., see Acernese

Franco, S., He, Y.-H., Sun, C. \& Xiao, Y., A comprehensive survey of brane tilings

Frasca, S., see Acernese

Frasconi, F., see Acernese

Frautschi, S., Stanley Mandelstam and my postdoctoral years at Berkeley

Frey, V., see Acernese

Froggatt, C. D., Nielsen, H. B., Nevzorov, R. \& Thomas, A. W., Dark energy density in SUGRA models and degenerate vacua

Fronsdal, C., Action principles for hydro- and thermo-dynamics

32 (2017) 1750061 Fu, H.-F., see Wang

Fucci, G., The Casimir effect for pistons with transmittal boundary

32 (2017) 1750202
32 (2017) 1730004

32 (2017) 1746010

32 (2017) 1750066

32 (2017) 1744003

32 (2017) 1750108

32 (2017) 1750079

32 (2017) 1744003

32 (2017) 1750119

32 (2017) 1744003

32 (2017) 1744003

32 (2017) 1730001

32 (2017) 1744003

32 (2017) 1744003

32 (2017) 1750078

32 (2017) 1744003

32 (2017) 1750015

32 (2017) 1747001

32 (2017) 1730020

32 (2017) 1744003

32 (2017) 1750142

32 (2017) 1744003

32 (2017) 1744003

32 (2017) 1740004

32 (2017) 1744003

32 (2017) 1730013

32 (2017) 1741016

32 (2017) 1750035

32 (2017) 1750182 
Fujikawa, K. \& Tureanu, A., Lorentz invariant $C P T$ breaking in the Dirac equation

Fukano, H. S., Top-mode pseudoNambu-Goldstone boson Higgs model

Fukushima, K. \& Sato, H., Toward construction of a consistent field theory with Poincaré covariance in terms of step-function-type basis functions for gauge fields

Gahramanov, I. \& Tezgin, K., A resurgence analysis for cubic and quartic anharmonic potentials

Gaillard, M. K., Remembering a gentle giant of physics

Gammaitoni, L., see Acernese

Gangopadhyay, S., see Banerjee

Gao, C., see Yang

Gao, J., see Bian

Gao, J., see Su

Gao, J., see Wang

Gao, J., see Wang

Gao, J., CEPC-SPPC accelerator status towards CDR

García Canal, C. A., see Fanchiotti

García-Aguilar, J. D. \& PérezLorenzana, A., Implications of Lorentz symmetry violation on a 5D supersymmetric model

Garufi, F., see Acernese

Gavrilov, S. P., see Adorno

Gemme, G., see Acernese

Geng, H., see Su

Geng, H., see Wang

Genin, E., see Acernese

Gennai, A., see Acernese

Georgi, H., Summary talk SCGT15

Germain, V., see Acernese

Ghasemkhani, M., see Bufalo

Ghezelbash, A. M. \& Kumar, V., Exact solutions to EinsteinMaxwell theory on EguchiHanson space

Ghorbani, K. \& Ghorbani, P. H., The LHC upper bounds for $p p \rightarrow$ diboson, $t \bar{t}$ cross-section on fermionic dark matter

Ghorbani, P. H., see Ghorbani

Ghosh, A., see Acernese

Ghosh, S., see Acernese

Ghosh, S., see Mitra

Giaccari, S. \& Nian, J., Dark solitons, $D$-branes and noncommuta- tive tachyon field theory

Giammarchi, M., Water purification in low background experiments

Giazotto, A., see Acernese

Giné, J., Inertial mass of an elemen-

32 (2017) 1747009 tary particle from the holographic scenario

Gitman, D. M., see Adorno

Giuliani, A., see Poda

Gnatenko, Kh. P. \& Tkachuk, V. M.,

32 (2017) $1730017 \quad$ Noncommutative phase space with rotational symmetry and hydrogen atom

32 (2017) 1750033 Goddard, P., The guiding influence of Stanley Mandelstam, from $S$ matrix theory to string theory

32 (2017) 1740016

32 (2017) 1744003

32 (2017) 1750115

32 (2017) 1746012

32 (2017) 1746009

32 (2017) 1746005

32 (2017) 1746006

32 (2017) 1746004

32 (2017) 1746003

32 (2017) 1750202

Goldman, T. \& Stephenson Jr., G. J., Implications of Higgs' universality for physics beyond the Standard Model

Gomez, J. D. \& Natale, A. A., QCD fixed points: Banks-Zaks scenario or dynamical gluon mass generation?

Gonzalez Castro, J. M., see Acernese

Gonzalez-Canales, F., see GómezIzquierdo

Gosselin, M., see Acernese

Goswami, J., Chakrabarti, D. \& Basak, S., Mass spectroscopy using Borici-Creutz fermions on 2D lattice

32 (2017) 1744003

32 (2017) 1750105

32 (2017) 1744003

32 (2017) 1746005

32 (2017) 1746006

32 (2017) 1744003

32 (2017) 1744003

32 (2017) 1747027

32 (2017) 1744003

32 (2017) 1750019

Gouaty, R., see Acernese

Grado, A., see Acernese

Granata, M., see Acernese

Grandou, T., see Bischer

Greco, G., see Acernese

Green, M. B., The influence of Stanley Mandelstam

Greselin, M., see Salesi

Grimus, W. \& Löschner, M., Revisiting on-shell renormalization conditions in theories with flavor mixing (Erratum)

32 (2017) 1750098

Groot, P., see Acernese

Gruning, P., see Acernese

Guidi, G. M., see Acernese

Gupta, S. \& Ruffo, S., The world of

32 (2017) 1750131 long-range interactions: A bird's

32 (2017) 1750131

32 (2017) 1744003

32 (2017) 1744003

32 (2017) 1750210 eye view

Gómez-Izquierdo, J. C., GonzalezCanales, F. \& Mondragón, M., On $\mathbf{Q}_{6}$ flavor symmetry and the breaking of $\mu \leftrightarrow \tau$ symmetry

Gómez-Valent, A., see Solà
32 (2017) 1750201

32 (2017) 1743011

32 (2017) 1744003

32 (2017) 1750043

32 (2017) 1750105

32 (2017) 1743012

32 (2017) 1750161

32 (2017) 1740013

32 (2017) 1742003

32 (2017) 1750012

32 (2017) 1744003

32 (2017) 1750171

32 (2017) 1744003

32 (2017) 1750059

32 (2017) 1744003

32 (2017) 1744003

32 (2017) 1744003

32 (2017) 1750118

32 (2017) 1744003

32 (2017) 1740018

32 (2017) 1750086

32 (2017) 1792001

32 (2017) 1744003

32 (2017) 1744003

32 (2017) 1744003

32 (2017) 1741018

32 (2017) 1750171

32 (2017) 1730014 
Hakimov, A., Abdujabbarov, A. \& Narzilloev, B., Quantum interference effects in conformal Weyl gravity

Han, C., see Dillon

Han, C., Probing light bino and higgsinos at the LHC

Harikumar, E. \& Zuhair, N. S., Hawking radiation in $\kappa$-spacetime

Harikumar, E., see Anjana

Harms, B., see Cavaglià

Harms, J., see Acernese

Hasenfratz, A., Brower, R. C., Rebbi, C., Weinberg, E. \& Witzel, O., Strongly coupled gauge theories: What can lattice calculations teach us?

Hayashii, H., see Bartoš

Haytmyradov, M., see $\mathrm{Hu}$

He, M., He, X.-G. \& Huang, C.-K., Dark photon search at a circular $e^{+} e^{-}$collider

He, X.-G., see He

He, Y.-H., see Franco

Heckman, J. J., Bernstein, J. G. \& Vigoda, B., MCMC with strings and Branes: the suburban algorithm (Extended Version)

Heidari, S., see Boroun

Heidmann, A., see Acernese

Heikinheimo, M., see Bernal

Heitmann, H., see Acernese

Hello, P., see Acernese

Hemming, G., see Acernese

Hewett, J. L., see Biekötter

Ho, C. M., see Edmonds

Hoak, D., see Acernese

Hofman, D., see Acernese

Hofmann, R., see Bischer

Holanda, O., see Anacleto

Holland, K., see Fodor

Hong, D. K., Holographic estimate of isospin splitting in hadron mass

Horikoshi, M., see Abe

Hosaka, A., see Jia

Hosseinkhani, H., Modarres, M. \& Olanj, N., Transverse momentum dependent (TMD) parton distribution functions generated in the modified DGLAP formalism based on the valence-like distributions

Hou, S., see Cavaglià

Houtz, R., see Abu-Ajamieh

Hoyos, C., see Alves
32 (2017) 1750116 32 (2017) 1745006

32 (2017) 1745003

32 (2017) 1750072

32 (2017) 1750183

32 (2017) 1750082

32 (2017) 1744003

32 (2017) 1747003 32 (2017) 1750154 32 (2017) 1730015

32 (2017) 1750138 32 (2017) 1750138 32 (2017) 1750142 Isac, J.-M., see Acernese

32 (2017) 1750133 32 (2017) 1750197 32 (2017) 1744003 32 (2017) 1730023 32 (2017) 1744003 32 (2017) 1744003 32 (2017) 1744003 32 (2017) 1750032 32 (2017) 1750108 32 (2017) 1744003 32 (2017) 1744003 32 (2017) 1750118 32 (2017) 1750128 32 (2017) 1747001

32 (2017) 1747013 32 (2017) 1750037 32 (2017) 1750153

32 (2017) 1750121 32 (2017) 1750082 32 (2017) 1750113 32 (2017) 1750200
Huet, D., see Acernese

Husain, T. Z., What remains invariant: Life lessons from Abdus Salam

Ianni, A., Review of technical features in underground laboratories Ibrahim, T., Itani, A., Nath, P. \& Zorik, A., Flavor violating top decays and flavor violating quark decays of the Higgs boson Incicchitti, A., see Bernabei Indumathi, D., see Saveetha Intini, G., see Acernese Ishii, M., see Miyahara Itani, A., see Ibrahim

Hu, Z., Liu, T., Leonardo, N. T Haytmyradov, M., Review of CMS

Itoh, K., Gauge symmetry and the functional renormalization group Itoyama, H., Ota, T., Suyama, T. \& Yoshioka, R., Cubic constraints for the resolvents of the ABJM matrix model and its cousins

Ittisamai, P., see Simmons

Iwazaki, A., Chiral symmetry breaking by monopole condensation

Iwazaki, A., QCD monopole and sigma meson coupling

J. Ambjørn \& Makeenko, Y., The use of Pauli-Villars regularization in string theory

Jacqmin, T., see Acernese

Jain, P., see Cadoni

Jaranowski, P., see Acernese

Jerusalimov, A. P. \& Lykasov, G. I., Proton distributions in $d p$ dielectron production within Regge theory

Jia, D., Pang, C.-Q. \& Hosaka, A., Mass formula for light nonstrange mesons and Regge trajectories in quark model

Jiang, Y., see Bian

Jiang, Y., see Wang

Jiang, Y., see Zhang
32 (2017) 1730015

32 (2017) 1750138

32 (2017) 1746012

32 (2017) 1730004

32 (2017) 1750014

32 (2017) 1746012

32 (2017) 1750204

32 (2017) 1750164

32 (2017) 1744003

32 (2017) 1741010

32 (2017) 1743001

32 (2017) 1750135

32 (2017) 1743007

32 (2017) 1750199

32 (2017) 1744003

32 (2017) 1744003

32 (2017) 1750205

32 (2017) 1750135

32 (2017) 1747011

32 (2017) 1750056

32 (2017) 1747023

32 (2017) 1750139

32 (2017) 1750109

32 (2017) 1750187

32 (2017) 1744003

32 (2017) 1750083

32 (2017) 1744003

32 (2017) 1750100

32 (2017) 1750153

32 (2017) 1746002

32 (2017) 1750035

32 (2017) 1750022 
Jing, J., see Wang

Jones, P., see Wulandari

Jonke, L., see Andrić

Jonker, R. J. G., see Acernese

Jung, S. \& Lee, H.-S., Constraining Higgsino kink tracks from existing LHC searches

Jurman, D., see Andrić

Järvinen, M., Holography and the conformal window in the Veneziano limit

Kabana, S. \& Minkowski, P., Counting of oscillatory modes of valence quarks forming $q q q$ baryons for three quark flavors $u, d, s$

Kadoh, D., Gauge/gravity duality From lattice gauge theory to black hole

Kahlhoefer, F., Review of LHC dark matter searches

Kakizaki, M., Santa, A. \& Seto, O., Phenomenological signatures of mixed complex scalar WIMP dark matter

Kalita, K., see Choudhury

Kaloshin, A. E. \& Lomov, V. P., On the polarization of fermion in an intermediate state

Kamani, D., see Maghsoodi

Kamani, D., see Saidy-Sarjoubi

Kapoor, A. K., see Anjana

Kashyap, S. P., see de Lacroix

Kato, S., see Kondo

Kato, S., see Shibata

Kawamura, Y., see Abe

Kawana, K., Possible explanations for fine-tuning of the universe

Khaidukov, Z. V. \& Zubkov, M. A., Modified top quark condensation model with the extra heavy fermion, the $125 \mathrm{GeV}$ pseudoGoldstone boson, and the additional heavy scalar bosons

Khalilov, V. R., Quantum states of a neutral massive fermion with an anomalous magnetic moment in an Aharonov-Casher field

Khan, I., see Acernese

Khanna, F. C., see Santos

Khanna, F. C., see Ulhoa

Khorramian, A., see Aleedaneshvar

Khorramian, A., see Rostami

Kim, J. S., see Biekötter

Kim, Y., see Lee

Kisslinger, L. S., Review of QCD,
32 (2017) 1750143 cosmological phase transitions,

32 (2017) $1750191 \quad$ QGP, heavy quark meson produc-

32 (2017) 1750046 tion enhancement and suppression

32 (2017) 1744003 Kitabayashi, T. \& Yasuè, M., Seesaw

model and two zero flavor neutrino texture

32 (2017) 1750070 Kitabayashi, T., Ohkawa, S. \& Yasuè,

32 (2017) $1750046 \quad$ M., One-loop radiative seesaw dark matter and neutrinoless double beta decay with two zero flavor neutrino mass texture

32 (2017) 1747017

Klauder, J. R., Mixed models: Combining incompatible scalar models in any space-time dimension

32 (2017) 1750004 Klinkhamer, F. R. \& Mistele, T., Classical stability of higherderivative $q$-theory in the fourform-field-strength realization

32 (2017) 1747018

32 (2017) 1730006

Kobakhidze, A., McDonald, K. L., Wu, L. \& Yue, J., Warped graviton couplings to bulk vectors with brane-localized kinetic terms

Kobakhidze, A., Exploring funda-

32 (2017) 1750038 mental physics with gravitational 32 (2017) 1750107 waves

Koch, P., Müller, B. \& Rafelski, J., From strangeness enhancement to 32 (2017) 1750096 quark-gluon plasma discovery 32 (2017) 1750063 Kodwani, D., see Baker

32 (2017) 1750069

32 (2017) 1750183

32 (2017) 1730021

32 (2017) 1747015

32 (2017) 1747016

32 (2017) 1750037

32 (2017) 1750048

Koide, Y. \& Nishiura, H., Flavon VEV scales in $\mathrm{U}(3) \times \mathrm{U}(3)^{\prime}$ model

Koley, S., see Acernese

Komal, B., see Yadav

Kondo, K.-I., Sasago, T., Shinohara, T., Shibata, A. \& Kato, S. Magnetic monopole versus vortex as gauge-invariant topological objects for quark confinement

Kondo, K.-I., see Shibata

Kong, E., see Wang

Koratzinos, M., see Bian

32 (2017) 1750141 Korchin, A. Yu., see Zagoskin

Kosasih, J. S., see Wulandari

Kouno, H., see Miyahara

Kowalska, I., see Acernese

32 (2017) 1750111 Kowalski-Glikman, J., A short intro32 (2017) 1744003 duction to $\kappa$-deformation

32 (2017) 1750132 Krishna, S., $\mathcal{N}=4$ supersymmet-

32 (2017) 1750094 ric quantum mechanical model: 32 (2017) $1750134 \quad$ Novel symmetries

32 (2017) 1750095 Kruglov, S. I., Inflation of universe 32 (2017) 1750032 due to nonlinear electrodynamics

32 (2017) 1750062 Kruglov, S. I., Magnetized black holes and nonlinear electro-
32 (2017) 1730008

32 (2017) 1750034

32 (2017) 1750186

32 (2017) 1750001

32 (2017) 1750090

32 (2017) 1745004

32 (2017) 1744001

32 (2017) 1730024

32 (2017) 1750198

32 (2017) 1750085

32 (2017) 1744003

32 (2017) 1750196

32 (2017) 1747015

32 (2017) 1747016

32 (2017) 1746008

32 (2017) 1746009

32 (2017) 1750166

32 (2017) 1750191

32 (2017) 1750205

32 (2017) 1744003

32 (2017) 1730026

32 (2017) 1750055

32 (2017) 1750071 
Cumulative author index

dynamics

Krämer, M., see Biekötter

Królak, A., see Acernese

Kuiroukidis, A., Inflationary $\alpha$ attractors and $F(R)$-gravity

Kuksa, V. I., see Bazhutov

Kuksa, V., see Beylin

Kumar, M., see Fang

Kumar, P., see Patgiri

Kumar, S., see Alam

Kumar, S., see Srinivas

Kumar, V., see Ghezelbash

Kunihiro, T., see Nonaka

Kurachi, M., see Aoki

Kurachi, M., see Aoki

Kureel, B. K., see Srinivas

Kuti, J., see Fodor

Kutynia, A., see Acernese

Kéfélian, F., see Acernese

Lartaux-Vollard, A., see Acernese

Laubenstein, M., Screening of materials with high purity germanium detectors at the Laboratori Nazionali del Gran Sasso

Lavrov, P. M., see Batalin

Lazzaro, C., see Acernese

Leaci, P., see Acernese

LeBohec, S., Scale relativistic signature in the Brownian motion of micro-spheres in optical traps

Lee, B.-H., Kim, Y., Pak, D. G., Tsukioka, T. \& Zhang, P. M., Gauge invariant gluon spin operator for spinless nonlinear wave solutions

Lee, H. M., see Dillon

Lee, H.-S., see Jung

Lee, S., Stanley Mandelstam: The early years at a "Most Stimulating Theoretical Group"

Leonardi, M., see Acernese

Leonardo, N. T., see $\mathrm{Hu}$

Leroy, N., see Acernese

Letendre, N., see Acernese

Li, Q., see Englert

Li, Q., see Wang

Li, Q., see Zhang

Li, T. G. F., Gravitational-wave observations from ground-based detectors

Li, T., Wang, W., Wang, X.-C. \& Xiong, Z.-H., The analytical oneloop contributions to Higgs boson mass in the Supersymmetric Standard Model with vector-like parti-
32 (2017) 1750147 cles

32 (2017) 1750032 Li, T., see Ding

32 (2017) 1744003 Li, X., Liu, X. \& Huang, Y.-C., Tackling tangledness of cosmic strings

32 (2017) 1750152 by knot polynomial topological in-

32 (2017) $1750188 \quad$ variants

32 (2017) 1750042 Li, Y., see Yang

32 (2017) 1746010 Li, Z.-Y., see Yu

32 (2017) 1750168 Lima, E. E. M., see Bazeia

32 (2017) 1750017 Lin, G.-L., see Chen

32 (2017) 1750136 Lin, Y.-H., see Chen

32 (2017) 1750098 Liu, G., see Peng

32 (2017) 1747014 Liu, J., see Yang

32 (2017) 1747005 Liu, T., see $\mathrm{Hu}$

32 (2017) 1747010 Liu, W., see Wu

32 (2017) 1750136 Liu, X., see Li

32 (2017) 1747001 Liu, Y., see Wu

32 (2017) 1744003 Lomov, V. P., see Kaloshin

32 (2017) 1744003 Long, C.-Y., see Wu

32 (2017) 1744003 Long, Z.-W., see Wang

Long, Z.-W., see $\mathrm{Wu}$

Loran, F., Chiral fermions on 2D curved space-times

32 (2017) 1743002 32 (2017) 1750189 32 (2017) 1744003 32 (2017) 1744003

32 (2017) 1750156 Lorenzini, M., see Acernese

Loriette, V., see Acernese

Losano, L., see Bazeia

Losurdo, G., see Acernese

Louzada, H. L. C. \& Belich, H., The Stark effect with minimum length

Lu, W., A Clifford algebra approach to chiral symmetry breaking and fermion mass hierarchies

Lucini, B., see Athenodorou

Lumaca, D., see Acernese

32 (2017) 1750062 32 (2017) 1745006 32 (2017) 1750070

32 (2017) 1740007 32 (2017) 1744003 32 (2017) 1730015 32 (2017) 1744003 32 (2017) 1744003 32 (2017) 1750106 32 (2017) 1750035 32 (2017) 1750022

32 (2017) 1744002 Luo, C.-B., Shi, S., Du, Y.-L., Wang, Y.-L. \& Zong, H.-S., Discussion on Lorentz invariance violation of noncommutative field theory and neutrino oscillation

Luo, X., see Fan

Lykasov, G. I., see Jerusalimov

Löschner, M., see Grimus

López-Sarrión, J., see Cortés

Maghsoodi, E. \& Kamani, D., Interaction of the branes in the presence of the background fields: The dynamical, nonintersecting, perpendicular, wrapped-fractional configuration

Majari, P., see Setare

Majhi, B. R., see Paul

Majhi, B. R., see Yadav

Maji, P., see Banerjee

Majorana, E., see Acernese

Makeenko, Y., see J. Ambjørn
32 (2017) 1745002

32 (2017) 1750014

32 (2017) 1750164

32 (2017) 1746012

32 (2017) 1750203

32 (2017) 1750163

32 (2017) 1750178

32 (2017) 1750178

32 (2017) 1750160

32 (2017) 1746012

32 (2017) 1730015

32 (2017) 1750125

32 (2017) 1750164

32 (2017) 1750148

32 (2017) 1750096

32 (2017) 1750148

32 (2017) 1750143

32 (2017) 1750148

32 (2017) 1750092

32 (2017) 1744003

32 (2017) 1744003

32 (2017) 1750163

32 (2017) 1744003

32 (2017) 1750010

32 (2017) 1750159

32 (2017) 1747006

32 (2017) 1744003

32 (2017) 1750040

32 (2017) 1750061

32 (2017) 1750100

32 (2017) 1792001

32 (2017) 1750084

32 (2017) 1750063

32 (2017) 1750158

32 (2017) 1750088

32 (2017) 1750196

32 (2017) 1750075

32 (2017) 1744003

32 (2017) 1750187 
Maksimovic, I., see Acernese

Malek, E., U-duality in three and four dimensions

Malik, R. P., see Srinivas

Malik, R. P., see Srinivas

Man, N., see Acernese

Mandal, T., see Marrani

Mantovani, M., see Acernese

Marchesoni, F., see Acernese

Marion, F., see Acernese

Mariotti, C. \& Passarino, G., Higgs boson couplings: Measurements and theoretical interpretation

Mariz, T., see Baeta Scarpelli

Markov, Yu. A., Markova, M. A. \& Bondarenko, A. I., Fourthorder wave equation in BhabhaMadhavarao spin- $\frac{3}{2}$ theory

Markova, M. A., see Markov

Marrani, A., Mandal, T. \& Tripathy, P. K., Supersymmetric black holes and Freudenthal duality

Marrani, A., Pradisi, G., Riccioni, F. \& Romano, L., Nonsupersymmetric magic theories and Ehlers truncations

Martelli, F., see Acernese

Martellini, L., see Acernese

Maru, N., Higgs mass in D-term triggered dynamical SUSY breaking

Maskawa, T., see Aoki

Maskawa, T., see Aoki

Masood, S. \& Saleem, I., Propagation of electromagnetic waves in extremely dense media

Masserot, A., see Acernese

Mastrogiovanni, S., see Acernese

Matsuzaki, S., One-family walking technicolor in light of LHC Run II

McDonald, K. L., see Kobakhidze

McKeon, D. G. C., see Brandt

Medina, A. D., see Cox

Mehraban, H., see Asadi

Mehraban, H., see Estabar

Mehri-Dehnavi, H., see Farahmand

Meidam, J., see Acernese

Meljanac, D., see Meljanac

Meljanac, S., Meljanac, D., Mignemi, S. \& Štrajn, R., Snyder-type space-times, twisted Poincaré algebra and addition of momenta

Mellado, B., see Fang

Mendizabal, S. \& Rojas, J. C., Backreaction effects on nonequilibrium spectral function
32 (2017) 1744003

32 (2017) 1750169

32 (2017) 1750136

32 (2017) 1750193

32 (2017) 1744003

32 (2017) 1750114

32 (2017) 1744003

32 (2017) 1744003

32 (2017) 1744003

32 (2017) 1730003

32 (2017) 1792002

32 (2017) 1750144

32 (2017) 1750144

32 (2017) 1750114

Mishra, R. \& Singh, H., Entangle-

32 (2017) 1750120

32 (2017) 1744003

32 (2017) 1744003

32 (2017) 1747020

32 (2017) 1747005

32 (2017) 1747010

32 (2017) 1750081

32 (2017) 1744003

32 (2017) 1744003

32 (2017) 1747004

32 (2017) 1745004

32 (2017) 1750003

32 (2017) 1750020

32 (2017) 1750110

32 (2017) 1750011

32 (2017) 1750066

32 (2017) 1744003

32 (2017) 1750172

32 (2017) 1750172

32 (2017) 1746010

32 (2017) 1750126 ment asymmetry for boosted black branes and the bound

Mistele, T., see Klinkhamer

Menon, M. J., see Fagundes

Merad, M., see Benchikha

Merzougui, M., see Acernese

Metzdorff, R., see Acernese

Meza-Aldama, O., see Arroyo-Ureña

Mezzani, F., see Acernese

Michel, C., see Acernese

Mignemi, S., see Meljanac

Milano, L., see Acernese

Milián, S. P., Supermultiplet of $\beta$ deformations from twistors

Miller, A., see Acernese

Milosevic, M., see Bilić

Minazzoli, O., see Acernese

Minenkov, Y., see Acernese

Minic, D., see Edmonds

Minkowski, P., see Kabana

Miramonti, L., Low background techniques in liquid scintillator detectors

Mirjalili, A., see Saffar

Mitra, A. K., Banerjee, R. \& Ghosh, S., On the equivalence among stress tensors in a gauge-fluid system

Mitra, A., Nonrelativistic fluids on scale covariant Newton-Cartan backgrounds

Miura, K., see Aoki

Miura, K., see Aoki

Miyahara, A., Ishii, M., Kouno, H. \& Yahiro, M., Crossover-model approach to QCD phase diagram, equation of state and susceptibilities in the $2+1$ and $2+1+1$ flavor systems

Mizoguchi, T., Biyajima, M. \& Suzuki, N., Analyses of whole transverse momentum distributions in $p \bar{p}$ and $p p$ collisions by using a modified version of Hagedorn's formula

Moazzen, M., Kalb-Ramond field localization in sine-Gordon braneworld models

Modarres, M., see Hosseinkhani

Moggi, A., see Acernese

Mohammadzadeh, H., see Farahmand

Mohan, M., see Acernese

Molgado, A., see Berra-Montiel
32 (2017) 1750184

32 (2017) 1750194

32 (2017) 1744003

32 (2017) 1744003

32 (2017) 1750195

32 (2017) 1744003

32 (2017) 1744003

32 (2017) 1750172

32 (2017) 1744003

32 (2017) 1750157

32 (2017) 1744003

32 (2017) 1750039

32 (2017) 1744003

32 (2017) 1744003

32 (2017) 1750108

32 (2017) 1750004

32 (2017) 1743010

32 (2017) 1750176

32 (2017) 1750091

32 (2017) 1750090

32 (2017) 1750210

32 (2017) 1750206

32 (2017) 1747005

32 (2017) 1747010

32 (2017) 1750205

32 (2017) 1750057

32 (2017) 1750058

32 (2017) 1750121

32 (2017) 1744003

32 (2017) 1750066

32 (2017) 1744003

32 (2017) 1750101 
Momeni-Feili, M., Arash, F., Taghavi-Shahri, F. \& Shahveh, A., Contribution of orbital angular momentum to the nucleon spin

Mondal, S., see Fodor

Mondragón, M., see GómezIzquierdo

Montani, M., see Acernese

Monteiro, A. P., Bhat, M. \& Vijaya Kumar, K. B., Mass spectra and decays of ground and orbitally excited $c \bar{b}$ states in nonrelativistic quark model

Montero, J. C., Pleitez, V., SánchezVega, B. L. \& Rodriguez, M. C., Supersymmetric $U(1)_{Y^{\prime}} \otimes$ $U(1)_{B-L}$ extension of the Standard Model

Morales, J., see Tarazona

Moretti, S., see Akeroyd

Mota, H. F., Bakke, K. \& Belich, H., Relativistic quantum scattering yielded by Lorentz symmetry breaking effects

Mottaghizadeh, M., Eslami, P. \& Taghavi-Shahri, F., Decoupling the NLO-coupled QED $\otimes$ QCD, DGLAP evolution equations, using Laplace transform method

Mounzi, Z., Safsafi, A., FerrichaAlami, M. \& Bennai, M., New constraint of an attractor GL model in braneworld context

Mours, B., see Acernese

Mukherjee, P., see Banerjee

Mukhopadhyay, S. \& Paul, C., Phases in holographic helical superconductor

Muroya, S., see Nonaka

Müller, B., see Koch

Márquez, F. \& Zamora, R., Critical end point in a thermomagnetic nonlocal NJL model

Márquez, F. \& Zamora, R., Thermomagnetic nonlocal NJL model in the real and imaginary time formalisms

Nagai, K.-i., see Aoki

Nagai, K.-i., see Aoki

Nagao, K. \& Nielsen, H. B., Momentum and Hamiltonian in complex action theory (Erratum)

Nakamura, A., see Nonaka

Nardecchia, I., see Acernese

Narzilloev, B., see Hakimov

Nascimento, J. R., see Baeta Scarpelli

32 (2017) 1792002

32 (2017) 1750036

Nasirimoghadam, S., see Azizi

Nastase, H., see Alves

Natale, A. A., see Gomez

Nath, P., see Ibrahim

32 (2017) 1750171

32 (2017) 1744003

Naticchioni, L., see Acernese

Nelemans, G., see Acernese

Neri, M., see Acernese

Neto, J. A., see Abreu

Neves, J. C. S., Deforming regular black holes

32 (2017) 1750021 Neves, M. J. \& Abreu, E. M. C., The standard electroweak model in the noncommutative DFR space-time

Neves, M. J., see Abreu

Nevzorov, R., see Froggatt

32 (2017) 1750093 32 (2017) 1750050 32 (2017) 1750145

g. W. K., see Ching

$\mathrm{Ng}$, Y. J., see Edmonds

Nian, J., see Giaccari

Nichols, D., see Acernese

Nielsen, H. B., see Andrić

Nielsen, H. B., see Froggatt

32 (2017) 1750140 Nielsen, H. B., see Nagao

Nikiforova, V., The stability of selfaccelerating universe in modified gravity with dynamical torsion

Nikkhoo, N. S. \& Shojaei, M. R.,

32 (2017) 1750065 Unpolarized and polarized densities based on a light-front quarkdiquark model

Nishiura, H., see Koide

32 (2017) 1750119 32 (2017) 1744003 32 (2017) 1750115

32 (2017) 1750064 32 (2017) 1747014 32 (2017) 1730024

32 (2017) 1750162

32 (2017) 1750027

32 (2017) 1747005 32 (2017) 1747010

32 (2017) 1792003

32 (2017) 1747014

32 (2017) 1744003

32 (2017) 1750116

Nisi, S., Copia, L., Dafinei, I. \& D Vacri, M. L., ICP-MS measurement of natural radioactivity at LNGS

Nissanke, S., see Acernese

Nocera, F., see Acernese

Nogradi, D., see Fodor

Nonaka, C., Kunihiro, T., Muroya, S., Nakamura, A., Sekiguchi, M. Wada, H. \& Wakayama, M., Scalar mesons in lattice QCD

Nunes, R. C., see Abreu

Nunes, R. C., see Oikonomou

Ohkawa, S., see Kitabayashi

Ohki, H., see Aoki

Ohki, H., see Aoki

Ohmi, K., Study of coherent beambeam instability in CEPC-H and Z

Ohya, S., Intertwining operator in thermal $\mathrm{CFT}_{d}$

Oikonomou, V. K., Pan, S. \& Nunes, R. C., Gravitational baryogenesis in running vacuum models
32 (2017) 1750177

32 (2017) 1750200

32 (2017) 1750012

32 (2017) 1750135

32 (2017) 1744003

32 (2017) 1744003

32 (2017) 1744003

32 (2017) 1750028

$32(2017) 1750112$

32 (2017) 1750190

32 (2017) 1750099

32 (2017) 1730013

32 (2017) 1750009

32 (2017) 1750108

32 (2017) 1750201

32 (2017) 1744003

32 (2017) 1750046

32 (2017) 1730013

32 (2017) 1792003

32 (2017) 1750137

32 (2017) 1750097

32 (2017) 1750085
32 (2017) 1743003

32 (2017) 1744003

32 (2017) 1744003

32 (2017) 1747001

32 (2017) 1747014

32 (2017) 1750028

32 (2017) 1750129

32 (2017) 1750186

32 (2017) 1747005

32 (2017) 1747010

32 (2017) 1746007

32 (2017) 1750006

32 (2017) 1750129 
Okorokov, V. A. \& Campos, S. D., Analysis of $p p$ and $\bar{p} p$ elastic scatterings based on theoretical bounds in high-energy physics: An update

Olanj, N., see Hosseinkhani

Olive, K. A., Supersymmetric versus $\mathrm{SO}(10)$ models of dark matter

Oota, T., see Itoyama

Osipov, A. A., see Volkov

Pak, D. G., see Lee

Pallante, E., Topology, the meson spectrum and the scalar glueball: Three probes of conformality and the way it is lost

Palomba, C., see Acernese

Pan, S., see Oikonomou

Pan, Z., Cui, Z.-F., Chang, C.H. \& Zong, H.-S., Finite-volume effects on phase transition in the Polyakov-loop extended NambuJona-lasinio model with a chiral chemical potential

Panda, B., see Banerjee

Pang, C.-Q., see Jia

Paoletti, F., see Acernese

Paoli, A., see Acernese

Park, M., see Dillon

Pasqualetti, A., see Acernese

Passaquieti, R., see Acernese

Passarino, G., see Mariotti

Passos, E., see Anacleto

Passuello, D., see Acernese

Patgiri, M., Kumar, P. \& Sarma, D., Study of texture zeros of fermion mass matrices in minimal extended seesaw mechanism and symmetry realization

Pati, J. C., Advantages of unity with SU(4)-color: Reflections through neutrino oscillations, baryogenesis and proton decay

Patricelli, B., see Acernese

Paul, A. \& Majhi, B. R., Hawking evaporation cascade in the presence of backreaction effect

Paul, C., see Mukhopadhyay

Pedurand, R., see Acernese

Pen, U.-L., see Baker

Peng, Y. \& Liu, G., Analytical studies on holographic superconductor in the probe limit

Perfilov, M., see Boos

Perreca, A., see Acernese

Peruzza, R. A., see Salesi
Petcov, S. T., On the IceCube result on $\bar{\nu}_{\mu} \rightarrow \bar{\nu}_{s}$ oscillations

Petrov, A. Yu., see Baeta Scarpelli

Piccinni, O. J., see Acernese

32 (2017) 1750175

32 (2017) 1750121

32 (2017) 1730010

32 (2017) 1750056

32 (2017) 1750123

32 (2017) 1750062

Pichot, M., see Acernese

Piergiovanni, F., see Acernese

Pillant, G., see Acernese

Pimentel, B. M., see Bufalo

Pinard, L., see Acernese

Pivovarov, A. A., see Volkov

Pleitez, V., see Montero

Poda, D. \& Giuliani, A., Low background techniques in bolometers for double-beta decay search

32 (2017) 1747025

32 (2017) 1744003

32 (2017) 1750129

Poggiani, R., see Acernese

Polchinski, J., Grad school with Stanley Mandelstam

Popolizio, P., see Acernese

Porter, E. K., see Acernese

Potter, C. T., NMSSM light decoupled Higgs singlet at CEPC

32 (2017) 1750067

32 (2017) 1750005

32 (2017) 1750153

32 (2017) 1744003

32 (2017) 1744003

32 (2017) 1745006

32 (2017) 1744003

32 (2017) 1744003

32 (2017) 1730003

32 (2017) 1750128

32 (2017) 1744003

Pourhassan, B., see Sadeghi

Pradisi, G., see Marrani

Preface

Prodi, G. A., see Acernese

Punturo, M., see Acernese

Puppo, P., see Acernese

Pérez-Lorenzana, A., see GarcíaAguilar

Quadri, A., Higgs potential from derivative interactions

Quevedo, F., ICTP: From a dream to a reality in 50+ years

Rabinovici, E., SESAME: A personal point of view

Rafelski, J., see Koch

32 (2017) 1750168

Ragab, N. A., see Zeng

Ramond, P., Mandelstam \& NAL

Randhawa, M., see Dahiya

Rapagnani, P., see Acernese

32 (2017) 1741013

32 (2017) 1744003

32 (2017) 1750088

32 (2017) 1750064

32 (2017) 1744003

32 (2017) 1750198

32 (2017) 1750160

32 (2017) 1750008

32 (2017) 1744003

32 (2017) 1750086

Rebhan, A. \& Turk, G., Polarization effects in light-by-light scattering: Euler-Heisenberg versus Born-Infeld
32 (2017) 1750018

32 (2017) 1792002

32 (2017) 1744003

32 (2017) 1744003

32 (2017) 1744003

32 (2017) 1744003

32 (2017) 1750165

32 (2017) 1744003

32 (2017) 1750123

32 (2017) 1750093

32 (2017) 1743012

32 (2017) 1744003

32 (2017) 1740005

32 (2017) 1744003

32 (2017) 1744003

32 (2017) 1746011

32 (2017) 1750045

32 (2017) 1750120

32 (2017) 1702002

32 (2017) 1744003

32 (2017) 1744003

32 (2017) 1744003

32 (2017) 1750052

32 (2017) 1750089

32 (2017) 1741003

32 (2017) 1741008

32 (2017) 1730024

32 (2017) 1750124

32 (2017) 1740017

32 (2017) 1750185

32 (2017) 1744003

32 (2017) 1750060

32 (2017) 1750047

32 (2017) 1750020

32 (2017) 1750045

32 (2017) 1744003

32 (2017) 1747003 
Rebhan, E., Model of a multiverse providing the dark energy of our universe

Regimbau, T., see Acernese

Rei, L., see Acernese

Ren, W., see Yang

Rezaei, B., see Boroun

Rho, M., The proton mass and scaleinvariant hidden local symmetry for compressed baryonic matter

Ricci, F., see Acernese

Riccioni, F., see Marrani

Rinaldi, E. (for the Lattice Strong Dynamics (LSD) Collaboration), Investigation of the scalar spectrum in $\mathrm{SU}(3)$ with eight degenerate flavors

Rinaldi, E., see Aoki

Rinaldi, E., see Aoki

Rizzo, T. G., see Biekötter

Robinet, F., see Acernese

Rocchi, A., see Acernese

Rodriguez, M. C., see Montero

Roenko, A. \& Sveshnikov, K., Perturbativity vs nonperturbativity in QED-effects for $\mathrm{H}$-like atoms with $Z \alpha>1$

Rojas, E., Covariant perturbations in the gonihedric string model

Rojas, J. C., see Mendizabal

Rolbiecki, K., see Biekötter

Rolland, L., see Acernese

Romano, L., see Marrani

Romano, R., see Acernese

Rosińska, D., see Acernese

Rostami, S. \& Khorramian, A., Trace of nonperturbative charm content of the nucleon on $Z+c$-jet production

Rouag, A., see Ydri

Ruffo, S., see Gupta

Ruggi, P., see Acernese

Sadeghi, J., Pourhassan, B., Tahery, S. \& Razavi, F., Holographic Schwinger effect with a deformed AdS background

Saffar, H. M., Mirjalili, A., Tehrani, S. A. \& Yazdanpanah, M. M., Sivers effect in single spin asymmetry based on the covariant parton model

Safsafi, A., see Mounzi

Sahoo, S., see Alam

Sahoo, S., see Banerjee

Saidy-Sarjoubi, M. \& Kamani, D.,
Interaction of dynamical fractional branes with background fields: Superstring calculations

32 (2017) 1750149 32 (2017) 1744003 32 (2017) 1744003 32 (2017) 1746012 32 (2017) 1750197 Saikia, B., see Choudhury Salam, A., Banquet speech at the Memorial Meeting for Abdus Salam's 90th Birthday

Salconi, L., see Acernese

Saleem, I., see Masood

32 (2017) 1747012 32 (2017) 1744003 32 (2017) 1750120

Salesi, G., Greselin, M., Deleidi, L. \& Peruzza, R. A., Modified Lorentz transformations in deformed special relativity

Santa, A., see Kakizaki

Santos, A. F. \& Khanna, F. C., Casimir effect for the Higgs field

32 (2017) 1747002 32 (2017) 1747005 32 (2017) 1747010 32 (2017) 1750032 32 (2017) 1744003 32 (2017) 1744003 32 (2017) 1750093 at finite temperature

Santos, A. F., see Ulhoa

Santos, W. O., Almeida, G. M. A. \& Souza, A. M. C., Noncommutative Brownian motion

Sarma, D., see Patgiri

Sasago, T., see Kondo

Sassolas, B., see Acernese

Sato, H., see Fukushima

Saveetha, H. \& Indumathi, D., Fragmentation of $\omega$ and $\phi$ mesons in $e^{+} e^{-}$and $p p$ collisions at NLO

32 (2017) 1750192 Schaich, D. \& Catterall, S., Maxi32 (2017) 1750126 mally supersymmetric Yang-Mills 32 (2017) 1750032 32 (2017) 1744003 32 (2017) 1750120 32 (2017) 1744003 32 (2017) 1744003 on the lattice

Schmidt, P., see Acernese

Schwarz, J. H., Reminiscences of Stanley Mandelstam

Sekiguchi, M., see Nonaka

Sen, A., see de Lacroix

Sentenac, D., see Acernese

Sepehri, A., Faizal, M., Setare, M. R.

32 (2017) 1750095 32 (2017) 1750180 32 (2017) 1741018 32 (2017) 1744003 \& Ali, A. F., Holographic cosmology from BIonic solutions

Sequino, V., see Acernese

Setare, M. R. \& Majari, P., The $(2+1)$-dimensional f-deformed Dirac oscillator in the presence of an external field

32 (2017) 1750045 Setare, M. R., see Sepehri

Seto, O., see Kakizaki

Shababi, H. \& Chung, W. S., Some quantum mechanical solutions in nonrelativistic anti-Snyder framework

32 (2017) 1750176 32 (2017) 1750119 32 (2017) 1750017 32 (2017) 1750075

Shafi, Q., Abdus Salam and Physics Beyond the Standard Model

Shahryari, Z., see Asadi

Shahveh, A., see Momeni-Feili
32 (2017) 1750069

32 (2017) 1750107

32 (2017) 1741009

32 (2017) 1744003

32 (2017) 1750081

32 (2017) 1750086 32 (2017) 1750038

32 (2017) 1750132 32 (2017) 1750094

32 (2017) 1750146

32 (2017) 1750168

32 (2017) 1747015

32 (2017) 1744003

32 (2017) 1730017

32 (2017) 1750199

32 (2017) 1747019

32 (2017) 1744003

32 (2017) 1740002

32 (2017) 1747014

32 (2017) 1730021

32 (2017) 1744003

32 (2017) 1750025

32 (2017) 1744003

32 (2017) 1750158 32 (2017) 1750025 32 (2017) 1750038

32 (2017) 1750170

32 (2017) 1741011

32 (2017) 1750110

32 (2017) 1750036 
Sharma, S., see Ahuja

She, Z.-L., see Zeng

Shi, S., see Luo

Shibata, A., Kondo, K.-I., Kato, S. \& Shinohara, T., Confinement/deconfinement phase transition in SU(3) Yang-Mills theory in view of dual superconductivity

Shibata, A., see Aoki

Shibata, A., see Aoki

Shibata, A., see Kondo

Shinohara, T., see Kondo

Shinohara, T., see Shibata

Shojaei, M. R., see Nikkhoo

Shrock, R., Recent results on renormalization-group evolution of theories with gauge, fermion, and scalar fields

Sidharth, B. G. \& Das, A., Comments on the paper "The zitterbewegung region"

Sidharth, B. G. \& Das, A., The zitterbewegung region

Sieniawska, M., see Acernese

Silva, P. V. R. G., see Fagundes

Simgen, H., see Wojcik

Simmons, E. H., Chivukula, R. S., Ittisamai, P. \& Vignaroli, N., Separating dijet resonances using the color discriminant variable

Simmons, E. H., see Chivukula Sin, S.-J., My advisor Stanley Singh, H., see Mishra

Singhal, A., see Acernese

Singleton, D., see Wulandari

Sivaramakrishnan, A., Colorkinematic duality in ABJM theory without amplitude relations

Skvortsov, E. D., see Bekaert

Smilga, A., Classical and quantum dynamics of higher-derivative systems

Solà, J., Gómez-Valent, A. \& de Cruz Pérez, J., Vacuum dynamics in the Universe versus a rigid $\Lambda=$ const.

Sommerfield, C., Remembering a gentle giant of physics

Sonnenschein, J., see Alves

Sorrentino, F., see Acernese

Soto, D. E., see Bufalo

Soudani, C., see Ydri

Souza, A. M. C., see Santos

Spannowsky, M., see Englert

Spray, A., see Cox
32 (2017) $1742005 \mid$ Srinivas, N. \& Malik, R. P., Nilpotent 32 (2017) 1750124 symmetries and Curci-Ferrari32 (2017) 1750040 type restrictions in 2D nonAbelian gauge theory: Superfield approach

Srinivas, N., Kumar, S., Kureel, B. K. \& Malik, R. P., Some novel features in 2D non-Abelian theory: BRST approach 32 (2017) 1747005 32 (2017) 1747010 32 (2017) 1747015 32 (2017) 1747015 32 (2017) 1747016 32 (2017) 1750097

32 (2017) 1747007

32 (2017) 1750173

32 (2017) 1750117

32 (2017) 1744003

32 (2017) 1750184

32 (2017) 1743004

32 (2017) 1747023

32 (2017) 1747022

32 (2017) 1740008

32 (2017) 1750091

32 (2017) 1744003

32 (2017) 1750191

32 (2017) 1750002 32 (2017) 1730019

32 (2017) 1730025

32 (2017) 1730014

32 (2017) 1740015 32 (2017) 1750200

32 (2017) 1744003 32 (2017) 1750165 32 (2017) 1750180 32 (2017) 1750146 32 (2017) 1750106 32 (2017) 1750020
Stelle, K. S., Abdus Salam and quadratic curvature gravity: Classical solutions

Stephenson Jr., G. J., see Goldman

Stratta, G., see Acernese

Su, F., Gao, J., Chen, Y., Tang, J., Wang, Y., Wang, D., Bai, S., Bian, T., Zhang, Y. \& Geng, H., SPPC/CEPC lattice design and beam dynamics study

Su, F., see Bian

Su, F., see Wang

Su, F., see Wang

Sugawara, H., Current algebra formulation of M-theory based on $E_{11}$ Kac-Moody algebra

Sugawara, H., High energy physics, past, present and future

Sun, C., see Franco

Sun, X., see Yang

Suyama, T., see Itoyama

Suzuki, N., see Mizoguchi

Sveshnikov, K., see Davydov

Sveshnikov, K., see Roenko

Swinkels, B. L., see Acernese

Sánchez-Vega, B. L., see Montero

Tacca, M., see Acernese

Taghavi-Shahri, F., see Momeni-Feili

Taghavi-Shahri, F., see Mottaghizadeh

Tahery, S., see Sadeghi

Takeuchi, T., see Edmonds

Talbert, J., see de Medeiros Varzielas

Talman, R., Ground-up circular Higgs Factory ring design and cell length optimization

Tang, H., see Wu

Tang, J., see Su

Tang, Y., Polynomial spectrum of gamma ray from dark matter

Tarazona, C. G., Diaz, R. A., Morales, J. \& Castillo, A., Phenomenology of the new physics coming from $2 \mathrm{HDMs}$ to the neutrino magnetic dipole moment

Tattersall, J., see Biekötter
32 (2017) 1750193

32 (2017) 1750136

32 (2017) 1741012

32 (2017) 1742003

32 (2017) 1744003

32 (2017) 1746005

32 (2017) 1746009

32 (2017) 1746006

32 (2017) 1746004

32 (2017) 1750024

32 (2017) 1741017

32 (2017) 1750142

32 (2017) 1746012

32 (2017) 1750056

32 (2017) 1750057

32 (2017) 1750054

32 (2017) 1750130

32 (2017) 1744003

32 (2017) 1750093

32 (2017) 1744003

32 (2017) 1750036

32 (2017) 1750065

32 (2017) 1750045

32 (2017) 1750108

32 (2017) 1750047

32 (2017) 1730005

32 (2017) 1750125

32 (2017) 1746005

32 (2017) 1745001

32 (2017) 1750050

32 (2017) 1750032 
Tavares-Velasco, G., see ArroyoUreña

Tehrani, S. A., see Saffar

Tenkanen, T., see Bernal

Tezgin, K., see Gahramanov

Thomas, A. W., see Froggatt

Thorn, C. B., Scientific biography of Stanley Mandelstam: 1955-1980

Ting, S., In Memory of Professor Kerson Huang

Tiwari, S., see Acernese

Tkachuk, V. M., see Gnatenko

Tokarev, M. V. \& Zborovský, I., New indication on scaling properties of strangeness production in $p p$ collisions at RHIC

Tonelli, M., see Acernese

Travasso, F., see Acernese

Tribelhorn, L., see Bornman

Trindade, M. A. S., see Floquet

Tringali, M. C., see Acernese

Tripathy, P. K., see Marrani

Triyanta, see Wulandari

Troshin, S. M. \& Tyurin, N. E., Experimental signatures of hadron asymptotics at the LHC

Trozzo, L., see Acernese

Tsang, K. W., see Acernese

Tsukioka, T., see Lee

Tumanov, A. G. \& West, P., $E_{11}$, Romans theory and higher level duality relations

Tuominen, K., see Bernal

Tuominen, K., Dynamical origin of the electroweak scale and a $125 \mathrm{GeV}$ boson

Tureanu, A., see Fujikawa

Turk, G., see Rebhan

Tyurin, N. E., see Troshin

Ulhoa, S. C., Santos, A. F. \& Khanna, F. C., Galilean covariance, Casimir effect and Stefan-Boltzmann law at finite temperature

Urrutia, L. F., see Escobar

van Bakel, N., see Acernese

van Beuzekom, M., see Acernese

van den Brand, J. F. J., see Acernese

Van Den Broeck, C., see Acernese

van der Schaaf, L., see Acernese

van Heijningen, J. V., see Acernese

Vardaro, M., see Acernese

Vaskonen, V., see Bernal

Veneziano, G., Remembering Stanley: From a source of inspiration to a fair strong competitor
Vento, V., see Fanchiotti

Vereshkov, G. M., see Bazhutov

Verma, M., see de Lacroix

32 (2017) 1750176

32 (2017) 1730023

32 (2017) 1750033

32 (2017) 1730013

32 (2017) 1740010

32 (2017) 1730009

32 (2017) 1744003

32 (2017) 1750161

32 (2017) 1750029

32 (2017) 1744003

32 (2017) 1744003

32 (2017) 1750150

32 (2017) 1750015

32 (2017) 1744003

32 (2017) 1750114

32 (2017) 1750191

32 (2017) 1750103

32 (2017) 1744003

32 (2017) 1744003

32 (2017) 1750062

32 (2017) 1750023

32 (2017) 1730023

32 (2017) 1747008

32 (2017) 1741014

32 (2017) 1750053

32 (2017) 1750103

32 (2017) 1750094

32 (2017) 1750077

32 (2017) 1744003

32 (2017) 1744003

32 (2017) 1744003

32 (2017) 1744003

32 (2017) 1744003

32 (2017) 1744003

32 (2017) 1744003

32 (2017) 1730023

32 (2017) 1740014
Verma, R., Quark mass matrices, textures and CKM precision measurements

Vianna, J. D. M., see Floquet

Vignaroli, N., see Chivukula

Vignaroli, N., see Simmons

Vigoda, B., see Heckman

Vijaya Kumar, K. B., see Monteiro

Vilela Mendes, R., A consistent measure for lattice Yang-Mills

Virasoro, M. A., Abdus Salam: The passionate, compassionate man and, his masterpiece, the ICTP

Volkas, R. R., Radiative neutrino mass generation: Models, flavour and the LHC

Volkov, M. K., Pivovarov, A. A. \& Osipov, A. A., The production of the $f_{1}(1285) \gamma$ and $a_{1}(1260) \gamma$ in colliding $e^{+} e^{-}$-beams in threshold domain

Voronina, Yu., see Davydov

Wada, H., see Nonaka

Wakayama, M., see Nonaka

Wang, B.-Q., see Wu

Wang, C., Kong, E., Cheng, D., Wang, Y., Zhang, K. \& Xu, Q., Electromagnetic design of a $12 \mathrm{~T}$ twin-aperture dipole magnet

Wang, D., Gao, J., Yu, C., Zhang, Y., Wang, Y., Su, F., Zhai, J., Bai, S., Geng, H., Bian, T., Wang, N., Cui, X. \& Zhang, C., 100 km CEPC parameters and lattice design

Wang, D., see Bian

Wang, D., see Su

Wang, F., Wang, W., Wu, L., Yang, J. M. \& Zhang, M., Probing degenerate heavy Higgs bosons in NMSSM with vector-like particles

Wang, G.-L., see Wang

Wang, G.-L., see Zhang

Wang, K., Zhang, Y.-F., Wang, Q., Long, Z.-W. \& Jing, J., Quantum speed limit for a relativistic electron in the noncommutative phase space

Wang, L., see Englert

Wang, M., see Englert

Wang, Q., see Wang
Wang, N., see Wang
32 (2017) 1750202

32 (2017) 1750188

32 (2017) 1730021

32 (2017) 1750151

32 (2017) 1750015

32 (2017) 1747022

32 (2017) 1747023

32 (2017) 1750133

32 (2017) 1750021

32 (2017) 1750016

32 (2017) 1741002

$32(2017) 1742001$

32 (2017) 1750123

32 (2017) 1750054

32 (2017) 1747014

32 (2017) 1747014

32 (2017) 1750148

32 (2017) 1746008

32 (2017) 1746006

32 (2017) 1746009

32 (2017) 1746005

32 (2017) 1745005

32 (2017) 1750035

32 (2017) 1750022

32 (2017) 1750143

32 (2017) 1750106

32 (2017) 1750106

32 (2017) 1746006

32 (2017) 1750143 
Wang, S.-W., Study of $\bar{B}_{s} \rightarrow$ $\phi \mu^{+} \mu^{-}$decays in the $G(211)$ model and vector leptoquark model

Wang, T., Fu, H.-F., Jiang, Y., Li, Q. \& Wang, G.-L., Annihilation rates of ${ }^{3} D_{2}\left(2^{--}\right)$and ${ }^{3} D_{3}\left(3^{--}\right)$ heavy quarkonia

Wang, T., see Zhang

Wang, W., see Li

Wang, W., see Wang

Wang, X.-C., see Li

Wang, Y., Su, F., Bai, S., Yu, C. \& Gao, J., Optics design for CEPC double ring scheme

Wang, Y., see Su

Wang, Y., see Wang

Wang, Y., see Wang

Wang, Y., Particle and astroparticle physics in China

Wang, Y.-L., see Luo

Wang, Y.-W., see Bian

Wang, Z.-G., see $\mathrm{Yu}$

Weber, T., see Biekötter

Weinberg, E., see Hasenfratz

West, P., see Tumanov

Witzel, O., see Hasenfratz

Wojcik, M., Zuzel, G. \& Simgen, H., Review of high-sensitivity Radon studies

Wong, C. H., see Fodor

Wu, B., Liu, W., Tang, H. \& Yue, R.-H., Destroying charged black holes in higher dimensions with test particles

Wu, L., see Kobakhidze

Wu, L., see Wang

Wu, S.-R., Long, Z.-W., Long, C.-Y., Wang, B.-Q. \& Liu, Y., $(2+1)$ dimensional Klein-Gordon oscillator under a magnetic field in the presence of a minimal length in the noncommutative space

Wulandari, D., Triyanta, Kosasih, J. S., Singleton, D. \& Jones, P., Localization of interacting fields in five-dimensional braneworld models

Xiao, L., see Yang

Xiao, Y., see Franco

Xiong, Z.-H., see Li

$\mathrm{Xu}, \mathrm{F}$., see Chen

$\mathrm{Xu}, \mathrm{Q} .$, see Wang

Yadav, G., Komal, B. \& Majhi, B. R.,

Rainbow Rindler metric and Un- ruh effect

Yagyu, K., see Akeroyd

Yahiro, M., see Miyahara

32 (2017) 1750155 Yamawaki, K., see Aoki

Yamawaki, K., see Aoki

Yamawaki, K., Old wine in a new bottle: Technidilaton as the $125 \mathrm{GeV}$ Higgs

32 (2017) 1750035 32 (2017) 1750022 32 (2017) 1745002 32 (2017) 1745005 32 (2017) 1745002

32 (2017) 1746004 32 (2017) 1746005 32 (2017) 1746008 32 (2017) 1746006

32 (2017) 1730027

32 (2017) 1750040 32 (2017) 1746009 32 (2017) 1750203 32 (2017) 1750032 32 (2017) 1747003 32 (2017) 1750023 32 (2017) 1747003

32 (2017) 1743004 32 (2017) 1747001

32 (2017) 1750125 32 (2017) 1745004 32 (2017) 1745005

32 (2017) 1750148

32 (2017) 1750191 32 (2017) 1746012 32 (2017) 1750142 32 (2017) 1745002 32 (2017) 1750178 32 (2017) 1746008

Yamazaki, T., see Aoki

Yamazaki, T., see Aoki

Yang, I-S., see Baker

Yang, J. M., see Wang

Yang, M. J. S., A symmetry breaking mechanism by parity assignment in the noncommutative Higgs model

Yang, P., Sun, X., Huang, G., Xiao, L., Gao, C., Huang, X., Zhou, W., Ren, W., Li, Y., Liu, J., You, B. \& Zhang, L., An asynchronous datadriven readout prototype for CEPC vertex detector

Yang, Q. \& Di, H., Axion-like particle dark matter in the linear regime of structure formation

Yasuè, M., see Kitabayashi

Yasuè, M., see Kitabayashi

Yazdanpanah, M. M., see Saffar

Ydri, B., Soudani, C. \& Rouag, A., Quantum gravity as a multitrace matrix model

Yeo, C. X., see Ching

Yildirim, E., see Akeroyd

Yoshimatsu, N., Implications of heavy neutralino dark matter: The underlying structure in the hidden sector

Yoshioka, R., see Itoyama

You, B., see Yang

Yu, C., see Wang

Yu, C., see Wang

Yu, G.-L., Wang, Z.-G. \& Li, Z.-Y., Analysis of the strong vertices of $\Sigma_{c}^{*} N D$ and $\Sigma_{b}^{*} N B$ in QCD sum rules

Yu, L., Salam's dream and dynamic changes in Chinese Condensed Matter Physics: A personal perspective

Yue, J., see Kobakhidze

Yue, R.-H., see Wu

Zagoskin, T. V. \& Korchin, A. Yu., The Higgs boson decay into $Z Z$ decaying to identical fermion pairs
32 (2017) 1750196

32 (2017) 1750145

32 (2017) 1750205

32 (2017) 1747005

32 (2017) 1747010

32 (2017) 1747026

32 (2017) 1747005

32 (2017) 1747010

32 (2017) 1750198

32 (2017) 1745005

32 (2017) 1747024

32 (2017) 1746012

32 (2017) 1750051

32 (2017) 1750186

32 (2017) 1750034

32 (2017) 1750176

32 (2017) 1750180

32 (2017) 1750009

32 (2017) 1750145

32 (2017) 1750013

32 (2017) 1750056

32 (2017) 1746012

32 (2017) 1746006

32 (2017) 1746004

32 (2017) 1750203

32 (2017) 1741007

32 (2017) 1745004

32 (2017) 1750125

32 (2017) 1750166 
Zamora, R., see Márquez

Zamora, R., see Márquez

Zborovský, I., see Tokarev

Zeng, T.-T., Chen, G., Dong, Z.J., She, Z.-L. \& Ragab, N. A., Study on fractal characteristics in the $e^{+} e^{-}$collisions at $\sqrt{s}=$ $250 \mathrm{GeV}$

Zhai, J., see Wang

Zhang, C., see Bian

Zhang, C., see Wang

Zhang, D.-X., see Chen

Zhang, J. \& Zhou, S., Neutrinos and cosmological matterantimatter asymmetry: A minimal seesaw with Frampton-GlashowYanagida ansatz

Zhang, K., see Wang

Zhang, L., see Yang

Zhang, M., see Wang

Zhang, P. M., see Lee

Zhang, S.-C., Wang, T., Jiang, Y., Li, Q. \& Wang, G.-L., Strong decays of $2^{+}$charm and charm-strange mesons

Zhang, Y., see Fang

\begin{tabular}{|c|c|}
\hline 32 (2017) 1750162 & Zhang, Y., see Su \\
\hline 32 (2017) 1750027 & Zhang, Y., see Wang \\
\hline \multirow[t]{4}{*}{32 (2017) 1750029} & Zhang, Y.-F., see Wang \\
\hline & $\begin{array}{l}\text { Zhao, Z.-H., On the breaking of } \mu-\tau \\
\text { permutation symmetry }\end{array}$ \\
\hline & Zheng, R., see Abu-Ajamieh \\
\hline & $\begin{array}{l}\text { Zheng, Z., Fang, S. \& Huang, G., } \tau \\
\text { mass and } R \text {-value measurements }\end{array}$ \\
\hline 32 (2017) 1746006 & at BES \\
\hline 32 (2017) 1746009 & Zheng, Z., Tsung-Dao Lee and \\
\hline 32 (2017) 1746006 & BEPC \\
\hline \multirow[t]{5}{*}{32 (2017) 1750207} & Zhou, S., see Zhang \\
\hline & Zhou, W., see Yang \\
\hline & Zhu, B., see Ding \\
\hline & Zhu, M., see Fang \\
\hline & $\begin{array}{l}\text { Zichichi, A., Abdus Salam, the elec- } \\
\text { troweak forces, ICTP and beyond }\end{array}$ \\
\hline 32 (2017) 1746008 & Zong, H.-S., see Fan \\
\hline 32 (2017) 1746012 & Zong, H.-S., see Luo \\
\hline 32 (2017) 1745005 & Zong, H.-S., see Pan \\
\hline \multirow[t]{4}{*}{32 (2017) 1750062} & Zorik, A., see Ibrahim \\
\hline & Zubkov, M. A., see Khaidukov \\
\hline & Zuhair, N. S., see Harikumar \\
\hline & Zuzel, G., see Wojcik \\
\hline 32 (2017) 1750022 & Štrajn, R., see Meljanac \\
\hline 32 (2017) 1746010 & \\
\hline
\end{tabular}

32 (2017) 1746005 32 (2017) 1746006 32 (2017) 1750143

32 (2017) 1742002

32 (2017) 1750113

32 (2017) 1730004

32 (2017) 1730007

32 (2017) 1742004

32 (2017) 1746012

32 (2017) 1750014

32 (2017) 1746010

32 (2017) 1741004

32 (2017) 1750061

32 (2017) 1750040

32 (2017) 1750067

32 (2017) 1750135

32 (2017) 1750141

32 (2017) 1750072

32 (2017) 1743004

32 (2017) 1750172 